\title{
Finite-N Conformality and Gauge Coupling Unification
}

\author{
Paul H. Frampton \\ Department of Physics and Astronomy, CB \#3255, University of North Carolina, Chapel Hill, NC \\ 27599-3255, USA.
}

\begin{abstract}
In this talk I review some aspects of the idea that there is an infra-red conformal fixedpoint at the TeV scale. In particular, it is shown how gauge coupling unification can be achieved by $\mathrm{TeV}$ unification in a semi-simple gauge group.
\end{abstract}

\section{INTRODUCTION}

Japan is the ideal place to talk about string theory since much of it originated here; for example, as a postdoc in Chicago my mentor Yoichiro Nambu explained to me in 1969(!) the relevance of two-dimensional conformal invariance.

In particle phenomenology, the impressive success of the standard theory based on $S U(3) \times S U(2) \times U(1)$ has naturally led to the question of how to extend the theory to higher energies? One is necessarily led by weaknesses and incompleteness in the standard theory. If one extrapolates the standard theory as it stands one finds (approximate) unification of the gauge couplings at $\sim 10^{16} \mathrm{GeV}$. But then there is the hierarchy problem of how to explain the occurrence of the tiny dimensionless ratio $\sim 10^{-14}$ of the weak scale to the unification scale. Inclusion of gravity leads to a super-hierarchy problem of the ratio of the weak scale to the Planck scale, $\sim 10^{18} \mathrm{GeV}$, an even tinier $\sim 10^{-16}$ Although this is obviously a very important problem about which conformality by itself is not informative, we shall discuss first the hierarchy rather than the superhierarchy.

There are four well-defined approaches to the hierarchy problem:

- 1. Supersymmetry

- 2. Technicolor.

- 3. Extra dimensions.

- 4. Conformality.

Supersymmetry has the advantage of rendering the hierarchy technically natural, that once the hierarchy is put in to the lagrangian it need not be retuned in perturbation theory. Supersymmetry predicts superpartners of all the known particles and these are predicted to be at or below a $\mathrm{TeV}$ scale if supersymmetry is related to the electroweak breaking. Inclusion of such hypothetical states improves the gauge coupling unification. On the negative side, supersymmetry does not explain the origin of the hierarchy. 
Technicolor postulates that the Higgs boson is a composite of fermion-antifermion bound by a new (technicolor) strong dynamics at or below the TeV scale. This obviates the hierarchy problem. On the minus side, no convincing simple model of technicolor has been found.

Extra dimensions can have a range as large as $1(\mathrm{TeV})^{-1}$ and the gauge coupling unification can happen quite differently than in only four spacetime dimensions. This replaces the hierarchy problem with a different fine-tuning question of why the extra dimension is restricted to a distance corresponding to the weak interaction scale.

Conformality is inspired by superstring duality and assumes that the particle spectrum of the standard model is enriched such that there is a conformal fixed point of the renormalization group at the $\mathrm{TeV}$ scale. Above this scale the coupling do not run so the hierarchy is nullified.

Conformality is the approach followed in this paper. We shall systematicaly analyse the compactification of the IIB superstring on $A d S_{5} \times S^{5} / \Gamma$ where $\Gamma$ is a discrete nonabelian group.

The duality between weak and strong coupling field theories and then between all the different superstring theories has led to a revolution in our understanding of strings. Equally profound, is the AdS/CFT duality which is the subject of the present article. This AdS/CFT duality is between string theory compactified on Anti-de-Sitter space and Conformal Field Theory.

Until very recently, the possibility of testing string theory seemed at best remote. The advent of $A d S / C F T$ s and large-scale string compactification suggest this point of view may be too pessimistic, since both could lead to $\sim 100 \mathrm{TeV}$ evidence for strings. With this thought in mind, we are encouraged to build $A d S / C F T$ models with realistic fermionic structure, and reduce to the standard model below $\sim 1 \mathrm{TeV}$.

Using AdS/CFT duality, one arrives at a class of gauge field theories of special recent interest. The simplest compactification of a ten-dimensional superstring on a product of an AdS space with a five-dimensional spherical manifold leads to an $\mathcal{N}=4 S U(N)$ supersymmetric gauge theory, well known to be conformally invariant[1]. By replacing the manifold $S^{5}$ by an orbifold $S^{5} / \Gamma$ one arrives at less supersymmetries corresponding to $\mathcal{N}=2,1$ or 0 depending [2] on whether $\Gamma \subset S U(2), S U(3)$, or $\not \subset S U$ (3) respectively, where $\Gamma$ is in all cases a subgroup of $S U(4) \sim S O(6)$ the isometry of the $S^{5}$ manifold.

It was conjectured in [3] that such $S U(N)$ gauge theories are conformal in the $N \rightarrow \infty$ limit. In [4] it was conjectured that at least a subset of the resultant nonsupersymmetric $\mathcal{N}=0$ theories are conformal even for finite $N$. Some first steps to check this idea were made in [5]. Model-building based on abelian $\Gamma$ was studied further in $[6,7,8]$, arriving in [8] at an $S U(3)^{7}$ model based on $\Gamma=Z_{7}$ which has three families of chiral fermions, a correct value for $\sin ^{2} \theta$ and a conformal scale $\sim 10 \mathrm{TeV}$.

The case of non-abelian orbifolds bases on non-abelian $\Gamma$ has not previously been studied, partially due to the fact that it is apparently somewhat more mathematically sophisticated. However, we shall show here that it can be handled equally as systematically as the abelian case and leads to richer structures and interesting results.

In such constructions, the cancellation of chiral anomalies in the four-dimensional theory, as is necessary in extension of the standard model (e.g. $[9,10])$, follows from 
the fact that the progenitor ten-dimensional superstring theory has cancelling hexagon anomaly[11]. It offers a novel approach to family unification[12, 13].

We consider all non-abelian discrete groups of order $g<32$. These are described in detail in $[14,15]$. There are exactly 45 such non-abelian groups. Because the gauge group arrived at by this construction[6] is $\otimes_{i} S U\left(N d_{i}\right)$ where $d_{i}$ are the dimensions of the irreducible representations of $\Gamma$, one can expect to arrive at models such as the PatiSalam $S U(4) \times S U(2) \times S U(2)$ type[16] by choosing $N=2$ and combining two singlets and a doublet in the 4 of $S U(4)$. Indeed we shall show that such an accommodation of the standard model is possible by using a non-abelian $\Gamma$.

The procedures for building a model within such a conformality approach are: (1) Choose $\Gamma$; (2) Choose a proper embedding $\Gamma \subset S U$ (4) by assigning the components of the 4 of $S U(4)$ to irreps of $\Gamma$, while at the same time ensuring that the 6 of $S U(4)$ is real; (3) Choose $N$, in the gauge group $\otimes_{i} S U\left(N d_{i}\right)$. (4) Analyse the patterns of spontaneous symmetry breaking.

In the present study we shall choose $N=2$ and aim at the gauge group $S U(4) \times$ $S U(2) \times S U(2)$. To obtain chiral fermions, it is necessary[6] that the $\mathbf{4}$ of $S U$ (4) be complex $\mathbf{4} \neq \mathbf{4}^{*}$. Actually this condition is not quite sufficient to ensure chirality in the present case because of the pseudoreality of $S U(2)$. We must ensure that the 4 is not just pseudoreal.

This last condition means that many of our 45 candidates for $\Gamma$ do not lead to chiral fermions. For example, $\Gamma=Q_{2 n} \subset S U$ (2) has irreps of appropriate dimensionalities for our purpose but it will not sustain chiral fermions under $S U(4) \times S U(2) \times S U(2)$ because these irreps are all, like $S U(2)$, pseudoreal. ${ }^{1}$ Applying the rule that 4 must be neither real nor pseudoreal leaves a total of only 19 possible non-abelian discrete groups of order $g \leq 31$. The smallest group which avoids pseudoreality has order $g=16$ but gives only two families. The technical details of our systematic search will be postponed to a future publication. Here we shall present only the simplest interesting non-abelian case which has $g=24$ and gives three chiral families in a Pati-Salam-type model[16].

Before proceeding to the details of the specific $g=24$ case, it is worth noting that the CFT it exemplifies should be free of all divergences if the conformality conjecture is correct and be UV finite. Further the theory is originating from a superstring theory in a higher-dimension (ten) and contains gravity[17, 18, 19] by compactification of the higher-dimensional graviton already contained in that superstring theory. In the CFT as we derive it in $\mathrm{d}=4$ flat spacetime, gravity is absent because we have not kept these graviton modes - of course, their influence on high-energy physics experiments is generally completely negligible unless the compactification scale is "large"[20].

To motivate our model it is instructive to comment on the choice of $\Gamma$ and on the choice of embedding.

If we embed only four singlets of $\Gamma$ in the 4 of $S U(4)$ then this has the effect of abelianizing $\Gamma$ and the gauge group obtained in the chiral sector of the theory is $S U(N)^{q}$. These cases can be interesting but have already been studied[6, 7]. Thus, we require at least one irrep of $\Gamma$ to have $d_{i} \geq 2$ in the embedding.

\footnotetext{
${ }^{1}$ Note that were we using $N \geq 3$ then a pseudoreal 4 would give chiral fermions.
} 
The only $\Gamma$ of order $g \leq 31$ with a 4 is $Z_{5} \tilde{\times} Z_{4}$ and this embedding leads to a nonchiral theory. This leaves only embeddings with two singlets and a doublet, a triplet and a singlet or two doublets.

The third of these choices leads to richer structures for low order $\Gamma$. Concentrating on them shows that of the chiral models possible, those from groups of low order result in an insufficient number (below three) of chiral families.

The first group that can lead to exactly three families occurs at order $g=24$ and is $\Gamma=Z_{3} \times Q$ where $Q\left(\equiv Q_{4}\right)$ is the group of unit quarternions which is the smallest dicyclic group $Q_{2 n}$.

There are several potential models due to the different choices for the 4 of $S U(4)$ but only the case $\mathbf{4}=\left(1 \alpha, 1^{\prime}, 2 \alpha\right)$ leads to three families so let us describe this in some detail:

Since $Q \times Z_{3}$ is a direct product group, we can write the irreps as $R_{i} \otimes \alpha^{a}$ where $R_{i}$ is a $Q$ irrep and $\alpha^{a}$ is a $Z_{3}$ irrep. We write $Q$ irreps as $1,1^{\prime}, 1^{\prime \prime}, 1^{\prime \prime \prime}, 2$ while the irreps of $Z_{3}$ are all singlets which we call $\alpha, \alpha^{2}, \alpha^{3}=1$. Thus $Q \times Z_{3}$ has fiveteen irreps in all and the gauge group will be of Pati-Salam type for $N=2$.

If we wish to break all supersymmetry, the $\mathbf{4}$ may not contain the trivial singlet of $\Gamma$. Due to permutational symmetry among the singlets it is sufficiently general to choose 4 $=\left(1 \alpha^{a_{1}}, 1^{\prime} \alpha^{a_{2}}, 2 \alpha^{a_{3}}\right)$ with $a_{1} \neq 0$.

To fix the $a_{i}$ we note that the scalar sector of the theory which is generated by the 6 of $S U(4)$ can be used as a constraint since the $\mathbf{6}$ is required to be real. This leads to $a_{1}+a_{2}=-2 a_{3}(\bmod 3)$. Up to permutations in the chiral fermion sector the most general choice is $a_{1}=a_{3}=+1$ and $a_{2}=0$. Hence our choice of embedding is

$$
\mathbf{4}=\left(1 \alpha, 1^{\prime}, 2 \alpha\right)
$$

with

$$
\mathbf{6}=\left(1^{\prime} \alpha, 2 \alpha, 2 \alpha^{2}, 1^{\prime} \alpha^{2}\right)
$$

which is real as required.

We are now in a position to summarize the particle content of the theory. The fermions are given by

$$
\sum_{I} 4 \times R_{I}
$$

where the $R_{I}$ are all the irreps of $\Gamma=Q \times Z_{3}$. This is:

$$
\begin{gathered}
\sum_{i=1}^{3}\left[\left(2{ }_{1} \alpha^{i}, 2_{2} \alpha^{i}\right)+\left(2{ }_{3} \alpha^{i}, 2_{4} \alpha^{i}\right)+\left(2{ }_{2} \alpha^{i}, 2{ }_{1} \alpha^{i}\right)+\left(2{ }_{4} \alpha^{i}, 2_{3} \alpha^{i}\right)+\left(4 \alpha^{i}, \overline{4} \alpha^{i}\right)\right] \\
+\sum_{i=1}^{3} \sum_{a=1}^{4}\left[\left(2{ }_{a} \alpha^{i}, 2_{a} \alpha^{i+1}\right)+\left(2_{a} \alpha^{i}, 4 \alpha^{i+1}\right)+\left(\overline{4} \alpha^{i}, 2_{a} \alpha^{i+1}\right)\right]
\end{gathered}
$$

It is convenient to represent the chiral portions of these in a given diagram (see Figure $1)$.

The scalars are given by

$$
\sum_{I} 6 \times R_{I}
$$


and are:

$$
\begin{gathered}
\sum_{i=1}^{3} \sum_{j=1(j \neq i)}^{3}\left[\left(2_{1} \alpha^{i}, 2_{2} \alpha^{j}\right)+\left(2{ }_{2} \alpha^{i}, 2{ }_{1} \alpha^{j}\right)+\left(2{ }_{3} \alpha^{i}, 2{ }_{4} \alpha^{j}\right)+\left(2{ }_{4} \alpha^{i}, 2{ }_{3} \alpha^{j}\right)+\left(2{ }_{2} \alpha^{i}, 2_{1} \alpha^{i}\right)+\left(2{ }_{4} \alpha^{i}, 2{ }_{3} \alpha^{i}\right)\right] \\
\left.+\sum_{i=1}^{3} \sum_{j=1(j \neq i)}^{3}\left\{\sum_{a=1}^{4}\left[\left(2{ }_{a} \alpha^{i}, 4 \alpha^{j}\right)+\overline{(4} \alpha^{i}, 2{ }_{a} \alpha^{j}\right)\right]+\left(4 \alpha^{i}, \overline{4} \alpha^{i}\right)\right\}
\end{gathered}
$$

which is easily checked to be real.

The gauge group $S U(4)^{3} \times S U(2)^{12}$ with chiral fermions of Eq.(4) and scalars of Eq.(6) is expected to acquire confromal invariance at an infra-red fixed point of the renormalization group, as discussed in [4].

To begin our examination of the symmetry breaking we first observe that if we break the three $S U(4)$ s to the totally diagonal $S U(4)$, then chirality in the fermionic sector is lost. To avoid this we break $S U_{1}(4)$ completely and then break $S U_{\alpha}(4) \times S U_{\alpha^{2}}$ (4) to its diagonal subgroup $S U_{D}(4)$. The first of these steps can be achieved with VEVs of the form $\left[\left(4_{1}, 2_{b} \alpha^{k}\right)+h . c\right.$.] where we are free to choose $b$, but $k$ must be 1 or 2 since there are no $\left(4_{1}, 2_{b} \alpha^{k=0}\right)$ scalars. The second step requires an

$S U_{D}(4)$ singlet VEV from $\left(\overline{4}_{\alpha}, 4_{\alpha^{2}}\right)$ and/or $\left(4_{\alpha}, \overline{4}_{\alpha^{2}}\right)$. Once we make a choice for $b$ (we take $b=4$ ), the remaining chiral fermions are, in an intuitive notation:

$$
\sum_{a=1}^{3}\left[\left(2_{a} \alpha, 1,4_{D}\right)+\left(1,2_{a} \alpha^{-1}, \overline{4_{D}}\right)\right]
$$

which has the same content as as a three family Pati-Salam model, though with a separate $S U_{L}(2) \times S U_{R}(2)$ per family.

To further reduce the symmetry we must arrange to break to a single $S U_{L}(2)$ and a single $S U_{R}(2)$. This is achieved by modifying step one where $S U_{1}(4)$ was broken. Consider the block diagonal decomposition of $S U_{1}(4)$ into $S U_{1 L}(2) \times S U_{1 R}(2)$. The representations $\left(2_{a} \alpha, 4_{1}\right)$ and $\left(2_{a} \alpha^{-1}, 4_{1}\right)$ then decompose as $\left(2_{a} \alpha, 4_{1}\right) \rightarrow\left(2_{a} \alpha, 2,1\right)+$ $\left(2_{a} \alpha, 1,2\right)$ and $\left(2_{a} \alpha^{-1}, 4_{1}\right) \rightarrow\left(2_{a} \alpha^{-1},, 2,1\right)+\left(2_{a} \alpha^{-1}, 1,2\right)$. Now if we give $V E V s$ of equal magnitude to the $\left(2_{a} \alpha, 2,1\right), a=1,2,3$, and equal magnitude $V E V s$ to the $\left(2_{a} \alpha^{-1}, 1,2\right) a=1,2,3$, we break $S U_{1 L}(2) \times \prod_{a=1}^{3} S U\left(2_{a} \alpha\right)$ to a single $S U_{L}(2)$ and we break $S U_{1 R}(2) \times \prod_{a=1}^{3} S U\left(2_{a} \alpha\right)$ to a single $S U_{R}(2)$. Finally, $V E V s$ for $\left(2{ }_{4} \alpha, 2,1\right)$ and $\left(2{ }_{4} \alpha, 1,2\right)$ as well as $\left(2{ }_{4} \alpha^{-1}, 2,1\right)$ and $\left(2{ }_{4} \alpha^{-1}, 1,2\right)$ insures that both $S U\left(2{ }_{4} \alpha\right)$ and $S U\left(2_{4} \alpha^{-1}\right)$ are broken and that only three families remain chiral. The final set of chiral fermions is then $3[(2,1,4)+(1,2, \overline{4})]$ with gauge symmetry $S U_{L}(2) \times S U_{R}(2) \times S U_{D}(4)$.

To achieve the final reduction to the standard model, an adjoint VEV from $\left(\overline{4}_{\alpha}, 4_{\alpha^{2}}\right)$ and/or $\left(4_{\alpha}, \overline{4}_{\alpha^{2}}\right)$ is used to break $S U_{D}(4)$ to the $S U(3) \times U(1)$, and a right handed doublet is used to break $S U_{R}(2)$.

While this completes our analysis of symmetry breaking, it is worthwhile noting the degree of constraint imposed on the symmetry and particle content of a model as the number of irreps $N_{R}$ of the discrete group $\Gamma$ associated with the choice of orbifold changes. The number of guage groups grows linearly in $N_{R}$, the number of scalar 
irreps grows roughly quadratically with $N_{R}$, and the chiral fermion content is highly $\Gamma$ dependent. If we require the minimal $\Gamma$ that is large enough for the model generated to contain the fermions of the standard model and have sufficient scalars to break the symmetry to that of the standard model, then $\Gamma=Q \times Z_{3}$ appears to be that minimal choice[21].

Although a decade ago the chances of testing string theory seemed at best remote, recent progress has given us hope that such tests may indeed be possible in AdS/CFTs. The model provided here demonstrates the standard model can be accomodated in these theories and suggests the possibility of a rich spectrum of new physics just around the $\mathrm{TeV}$ corner.

\section{GAUGE COUPLING UNIFICATION}

There is not space here to describe many technical details which are, however, available in the published papers cited at the end of this talk. But I would like to emphasize one success of the approach which involves the unification of gauge couplings [7, 22]. Recall that the successful such unification is one primary reason for belief in supersymmetric grand unification e.g. [23]. That argument is simple to state: The RG equations are:

$$
\frac{1}{\alpha_{i}\left(M_{G}\right)}=\frac{1}{\alpha_{i}\left(M_{Z}\right)}-\frac{b_{i}}{2 \pi} \ln \left(\frac{M_{G}}{M_{Z}}\right)
$$

Using the LEP values at the Z-pole as $\alpha_{3}=0.118 \pm 0.003, \alpha_{2}=0.0338$ and $\alpha_{1}=$ $\frac{5}{3} \alpha_{Y}=0.0169$ (where the eroors on $\alpha_{1,2}$ are less than $1 \%$ ) and the MSSM values $b_{i}=\left(6 \frac{3}{5}, 1,-3\right)$ leades to $M_{G}=2.4 \times 10^{16} \mathrm{GeV}$ and the predictiion that $\sin ^{2} \theta=0.231$ in excellent agreement with experiment.

In the present approach the three gauge couplings $\alpha_{1,2,3}$ run up to $\sim 1 \mathrm{TeV}$ where they freeze and embed in a larger (semi-simple) gauge group which contains $S U(3) \times$ $S U(2) \times U(1)$.

I will give two examples, the first based on the abelian orbifold $S^{5} / Z_{7}$ and the second based on the non-abelian orbifold $S^{5} /\left(D_{4} \times Z_{3}\right)$.

In the first, abelian, case we choose $\mathrm{N}=3, \Gamma=Z_{7}$ and the unifying group is therefore $S U(3)^{7}[7,8]$. It is natural to accommodate one $S U(3)$ factor (color) into one of the seven $S U$ (3) factors, $S U(2)_{L}$ as a diagonal subgroup of two and to identify the correctly normalized $U(1)$ as the diagonal subgroup of the remaining four $S U(3)$ factors. This implies that $\alpha_{2} / \alpha_{1}=2$ and consequently:

$$
\sin ^{2} \theta=\frac{\alpha_{Y}}{\alpha_{2}+\alpha_{Y}}=\frac{3 / 5}{2+3 / 5}=\frac{3}{13}=0.231
$$

There is a small correction for the running between $M_{Z}$ and the TeV scale but this is largely compensated by the two-loop correction and the agreement remains as good as for SUSY-GUTS. This is strong encouragement for the conformality approach. 
In the second, non-abelian, example we use $\Gamma=Z_{3} \times D_{4}$ and choose $\mathrm{N}=2$ to arrive at a unification based on the Pati-Salam group $S U(4)_{C} \times S U(2)_{L} \times S U(2)_{R}$ instead of the trinification $S U(3)^{3}$. This is possible because this non-abelian $\Gamma$ has two-dimensional representations as well as one-dimensional ones.

By the way, the dihedral group $D_{4}$ consists of eight rotations which leave a square invariant: two of the rotations are flips about two lines which bisect the square and the other four are rotations through $\pi / 2, \pi, 3 \pi / 2$ and $2 \pi$ about the perpendicular to the plane of the square.

In this case the low energy gauge group is thus $S U(4)^{3} \times S U(2)^{12}$. We embed $S U(3)_{\text {color }}$ in $\mathrm{r}$ of the $S U(4)$ groups where $\mathrm{r}=1$ or 2 because $\mathrm{r}=3$ leads to loss of chirality. At the same time the $S U(2)_{L}$ and $S U(2)_{R}$ are respectively embedded in diagonal subgroups of $\mathrm{p}$ and $\mathrm{q}$ of the twelve $S U(2)$ factors where $\mathrm{p}+\mathrm{q}=12$.

Since $\mathrm{p}$ and $\mathrm{q}$ are necessarily integers it is not at all obvious a priori that the value of $\sin ^{2} \theta$ can be consistent with experiment.

The values of the respective couplings at the conformality/unification scale are now:

$$
\begin{gathered}
\alpha_{2 L}^{-1}\left(M_{U}\right)=p \alpha_{U}^{-1} \\
\alpha_{2 R}^{-1}\left(M_{U}\right)=q \alpha_{U}^{-1} \\
\alpha_{4 C}^{-1}\left(M_{U}\right)=2 r \alpha_{U}^{-1}
\end{gathered}
$$

The hypercharge coupling is related by

$$
\alpha_{1}^{-1}=\frac{2}{5} \alpha_{4 C}^{-1}+\frac{3}{5} \alpha_{2 R}^{-1}
$$

Defining $y=\ln \left(M_{U} / M_{Z}\right)$ we then find the general expression for $\sin ^{2} \theta_{W}\left(M_{Z}\right)$ to be:

$$
\sin ^{2} \theta_{W}\left(M_{Z}\right)=\frac{p-(19 / 12 \pi) y \alpha_{U}}{p+q+\frac{4}{3} r+(11 / 6 \pi) y \alpha_{U}}
$$

Here

$$
\alpha_{S}^{-1}\left(M_{Z}\right)=2 r \alpha_{U}^{-1}-\frac{7}{2 \pi} y
$$

Using these formulas and $\alpha_{S}\left(M_{Z}\right) \sim 0.12$ we find for the natural choices (for model building) $\mathrm{p}=4$ and $\mathrm{r}=2$ that

$$
\sin ^{2} \theta_{W}\left(M_{Z}\right) \simeq 0.23
$$

again in excellent agreement with experiment.

It is highly non-trivial that again the gauge coupling unification works in this case which, according to the lengthy analysis in the second paper of [21], is the unique accommodation of the standard model with three chiral families for all non-abelian $\Gamma$ with order $g \leq 31$. 


\section{DISCUSSION}

The successful derivation of $\sin ^{2} \theta_{W}\left(M_{Z}\right) \simeq 0.23$ from both the abelian orbifold (based on 333-trinification) and the non-abelian orbifold (based on 422-Pati-Salam unification) is strong support for further investigation of the detailed phenomenology arising from the approach.

More generally, the conformality provides a rigid organizing principle which strongly constrains all couplings and parameters in the low-energy lagrangian. Breaking of conformal symmetry clearly needs much more study: so far, soft breaking has been considered mainly because is is technically easier but spontaneous breaking would be more satisfactory. One can even speculate that nonsupersymmetric $\mathcal{N}=0$ theories could have a "flat" direction. After all, if any such theory is finite in the UV, it likely possesses non-renormalization theorems derivable from a symmetry different from global supersymmetry.

In any case, a conformal IR fixed point at the TeV scale necessitates new particles (of spins $1,1 / 2$ and 0 ) which await discovery in that energy regime.

\section{ACKNOWLEDGEMENTS}

The organizers must be thanked for creating such an excellent Symposium. This work was supported in part by the US Department of Energy under Grant No. DE-FG0297ER-41036. 


\section{REFERENCES}

1. S. Mandelstam, Nucl. Phys. B213, 149 (1983).

2. S. Kachru and E. Silverstein, Phys. Rev. Lett. 80, 4855 (1998).

3. J. Maldacena, Adv. Theor. Math. Phys. 2, 231 (1998).

4. P.H. Frampton, Phys. Rev. D60, 041901 (1999).

5. P.H. Frampton and W. F. Shively, Phys. Lett. B454, 49 (1999).

6. P.H. Frampton and C. Vafa, hep-th/9903226.

7. P.H. Frampton, Phys. Rev. D60, 085004 (1999).

8. P.H. Frampton, Phys. Rev. D60, 121901 (1999).

9. P.H. Frampton and S.L. Glashow, Phys. Lett. 190B, 157 (1987); Phys. Rev. Lett. 58, 2168 (1987).

10. P.H. Frampton, Phys. Rev. Lett. 69, 2889 (1992).

11. P.H. Frampton and T.W. Kephart, Phys. Rev. Lett. 50, 1343 (1983); ibid 50, 1347 (1983).

12. Another (non-minimal) approach to family symmetry appeared in: P.H. Frampton, Phys. Lett. B88, 299 (1979).

13. S. Pakvasa and H. Sugawara, Phys. Lett. B73, 61 (1978).

14. Useful sources of information on the finite groups include:

D.E. Littlewood, it The Theory of Group Characters and Matrix Representations of Groups (Oxford 1940);

M. Hamermesh, Group Theory and Its Applications to Physical Problems (Addison-Wesley, 1962); J.S. Lomont, Applications of Fimite Groups (Academic, 1959), reprinted by Dover (1993); A.D. Thomas and G.V. Wood, Group Tables (Shiva, 1980).

15. P.H. Frampton and T.W. Kephart, Int. J. Mod. Phys. A10, 4689 (1995).

16. J.C. Pati and A. Salam, Phys. Rev. D10, 275 (1974).

R.N. Mohapatra and J.C. Pati, Phys. Rev. D11, 566 (1975).

R.N. Mohapatra and G. Senjanovic, Phys. Rev. D12, 1502 (1975)

17. H. Verlinde, Nucl. Phys. B580, 264 (2000).

18. L. Randall and R. Sundrum, Phys. Rev. Lett. 833370 (1999); ibid 83, 4690 (1999).

J. Lykken and L. Randall, JHEP 0006 (2000) 014.

19. W.D. Goldberger and M. B. Wise, Phys. Rev. D60, 107505 (1999); Phys. Rev. Lett. 83, 4922 (1999); Phys. Lett. B475, 275 (2000).

20. I. Antoniadis, Phys. Lett. B246 (1990) 377;

I. Antoniadis and K. Benakli, Phys. Lett. B326 (1994) 69;

I. Antoniadis, K. Benakli and M. Quiros, Phys. Lett. B331 (1994) 313.

J. D. Lykken, Phys. Rev. D54 (1996) 3693.

I. Antoniadis et al, Phys. Lett. B436, 257 (1998).

I. Antoniadis, S. Dimopoulos, A. Pomarol and M. Quiros, Nucl. Phys. B544, 503 (1999).

K. Dienes, E. Dudas and T. Gherghetta, Phys. Lett. B436 (1998) 55; Nucl. Phys. 537, 47 (1999); Nucl. Phys. B543, 387 (1999).

P.H. Frampton and A. Rasin, Phys. Lett. 460B, 313 (1999).

D. Ghilencea and G.G. Ross, Phys. Lett. B442 (1998) 165.

C.D. Carone, Phys. Lett. B454, 70 (1999).

C. Bachas, JHEP 981, 023 (1998).

G. Shiu and S.H.H. Tye, Phys. Rev. D58 (1998) 106007.

Z. Kakushadze and S.H.H. Tye, Nucl. Phys. B548, 180 (1999).

Z. Kakushadze, Nucl. Phys. B551, 549 (1999).

N. Arkani-Hamed, S. Dimopoulos and G. Dvali, Phys. Lett 429 (1998)506.

21. P.H. Frampton and T.W. Kephart, Phys. Lett. B485, 403 (2000) and Phys. Rev. D (2001, in press) hep-th/0011186.

22. P.H. Frampton, R.N. Mohapatra and S. Suh. hep-ph/0104211. submitted to Phys. Lett. B.

23. S. Dimopoulos, S. Raby and F. Wilczek, Phys. Rev. D24, 1681 (1981).

N. Sakai, Z. Phys C11, 153 (1981).

U. Amaldi, W. De Boer and H. Furstenau, Phys. Lett. B260, 447 (1991).

U. Amaldi, W. De Boer, P.H. Frampton, H. Furstenau and J.T. Liu, Phys. Lett. B281, 374 (1992). 